\title{
Harnessing Endogenous T-Regulatory Cells in Acute Kidney Injury
}

\author{
Rahul Sharma
}

Center for Immunity, Inflammation and Regenerative Medicine, Division of Nephrology, Department of Medicine, University of Virginia, Charlottesville, VA, USA

\section{Keywords}

T-regulatory cells · Acute kidney injury · Interleukin-2 · Interleukin-33 · Inflammation · Regeneration

\begin{abstract}
Acute kidney injury (AKI) affects $20-50 \%$ of hospitalized and intensive care patients, with a sizable proportion progressing to ESRD or death (https://www.kidney.org/atoz/content/AcuteKidneylnjury). Since inflammation contributes to the pathogenesis of AKI, studies on T-regulatory cells (Tregs), which suppress inflammation are important. Here, we highlight advances that utilize Tregs for intervention in AKI and were presented at the 25th International Conference on Advances in Critical Care Nephrology and UAB/UCSD O'Brien Center AKI \& CRRT 2020.

๑) 2020 S. Karger AG, Basel
\end{abstract}

\section{Introduction}

T-regulatory cells (Tregs) are a subset of helper T-cells that are identified by the expression of interleukin (IL)2Ra (CD25) and the Forkhead BoxP3 (Foxp3) transcription factor (reviewed in [1]). Tregs are one of the major mechanisms for immune tolerance and preventing abnormal activation of immune system. Mutations in IL-2/ IL-2R or Foxp3 induce Treg-deficiency and fatal autoim- mune inflammation. Many cell-surface and intracellular molecules have been identified that are either expressed on Tregs and regulate their survival and function [1]. Acute kidney injury (AKI) occurs due to diverse conditions, including ischemia, sepsis, nephrotoxicity, transplantation, etc., and is exacerbated by inflammation [1].

\section{Tregs in AKI}

A role of Tregs in AKI was first identified when depletion of Tregs with an anti-CD25 antibody, administered before ischemia-reperfusion injury (IRI), increased the susceptibility of mice to inflammation and injury [2]. Tregs were found to persist in the kidneys up to ten days post-IRI [3]. Depletion of Treg one day after IRI also worsened mortality and delayed recovery [3]. Increasing Treg numbers by infusion of Tregs isolated from donor wild-type mice either before or after IRI increased the resistance of the recipient mice to AKI [2] or improved recovery, respectively [3]. Lower expression of TNFRII or

Contribution from the AKI and CRRT 2020 Symposium at the 25th International Conference on Advances in Critical Care Nephrology, Manchester Grand Hyatt, San Diego, CA, USA, February 24-27th, 2020. This symposium was supported in part by the NIDDK funded University of Alabama at Birmingham-University of California San Diego O'Brien Center for Acute Kidney Injury Research (P30DK079337). karger@karger.com www.karger.com/nef

(c) 2020 S. Karger AG, Basel

Karger"
Dr. Rahul Sharma

Center for Immunity Inflammation and Regenerative Medicine Division of Nephrology, Department of Medicine, University of Virginia P.O. Box 800133, Charlottesville, VA 22908 (USA)

rs3wn@hscmail.mcc.virginia.edu 
Fig. 1. Regulatory T-cells (Tregs) prevent the progression of AKI by restricting inflammation and also promote repair and regeneration. Proliferation, activation, or stabilization of endogenous Tregs by IL-2C (IL-2/anti-IL-2 Ab complex) or a novel hybrid cytokine IL233 (bearing IL-2 and IL33 in a single molecule) is strongly protective. Conversion of ATP to adenosine by CD39 and CD73 and its (or analog ATL1222) utilization through adenosine receptor $\left(\mathrm{A}_{2 \mathrm{~A}} \mathrm{R}\right)$ can promote Tregs through $\mathrm{PD}-1$, which may enhance Treg metabolism through mTOR. Other mediators that promote endogenous Tregs in the settings of AKI include, but are not limited to, sphingosine receptor (S1P1) agonist FTY720, rapamycin, $\mathrm{P} 2 \mathrm{X} 7$ antagonist periodate-oxidized ATP (oATP), or bee-venom phospholipase A2 (bvPLA2) that works through dendritic cells. Tregs, T-regulatory cells; AKI, acute kidney injury.

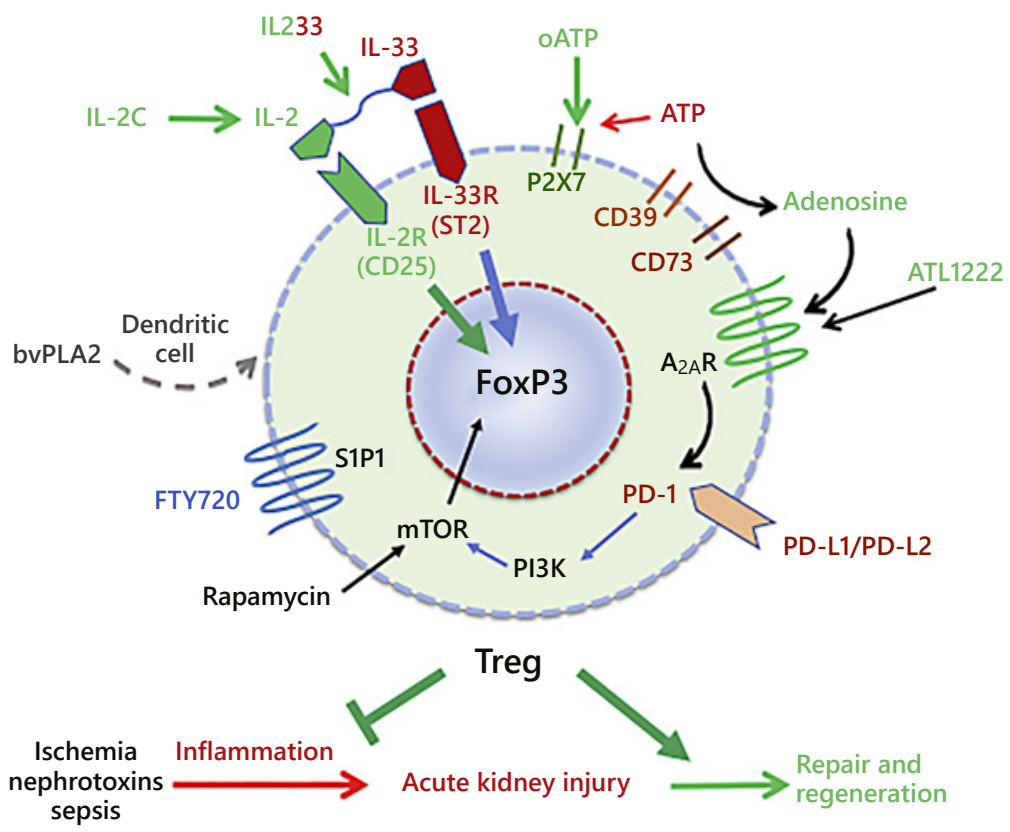

sepsis reduced suppressive activity of circulating Tregs pretransplantation correlated with delayed graft function and AKI [4]. Since AKI is frequent in cardiac surgery patients, we evaluated the levels of circulating Tregs in these patients before surgery. Indeed, the numbers of circulating Tregs prior to cardiac surgery could be a predictor of AKI as they negatively correlated with the incidence of AKI (Rahul Sharma, Gilbert Kinsey, Murat Dogan, and Mitchell Rosner, manuscript under preparation).

ATP released during cellular damage acts as a danger signal and is usually converted by a pair of ectonucleotidases CD39 and CD73 to the anti-inflammatory adenosine. In adoptive transfer studies, Tregs from CD39 overexpressing mice were more protective in IRI than those from wild-type mice [5]. On the other hand, donor Tregs from mice deficient in $\mathrm{CD} 73$ or adenosine receptor (A2AR) failed to offer any protection [6]. Ex vivo treatment of Tregs with ATL1222, a synthetic agonist of A2AR increased the expression of the checkpoint inhibitor PD-1 on Tregs and enhanced their ability of Tregs to protect in IRI [6]. Injection of blocking antibodies to either ligands of PD-1 (PD-L1/L2) negated the ability of Tregs to protect from IRI [7]. Further, Tregs from PD-1-deficient mice are also unable to protect mice from IRI in adoptive transfer studies and may regulate the function of Tregs through mTOR signaling (Gilbert Kinsey, Murat Dogan, and Rahul Sharma, manuscript under preparation). Indeed, treatment with the mTOR-inhibitor rapamycin enhanced the protective activity of Tregs in IRI [8]. Thus, Treg-mediated protection from AKI involves conversion of ATP to adenosine and its autocrine utilization, which promotes PD-1 on Tregs to stimulate mTOR signaling (Fig. 1). Enhancing Treg number or function may be a therapeutic target for intervention in AKI.

\section{Therapeutic Approaches Targeting Endogenous Tregs}

Although autologous Treg therapy is being explored in autoimmune diseases (NCT01210664 and NCT02428309), it may not be suitable for AKI because ex vivo expansion of autologous Tregs to required numbers takes several weeks, and there are still doubts of their specificity and stability. Thus, approaches that could enhance endogenous Tregs are highly desirable. Some strategies that indirectly increased the levels of Tregs and found to be protective in AKI include ischemic preconditioning [9] or pharmacologic treatment with the sphingosine-1-phosphate analog FTY720 [10], phospholipase $\mathrm{A} 2$ [11], or the purinergic $\mathrm{P}_{2} \mathrm{X}_{7}$ antagonist periodate-oxidized ATP [12]. Among some of the approaches that can 
directly act on Tregs, treatment with an IL-2/anti-IL-2 antibody complex robustly increased Tregs in the spleen and kidneys, as well as attenuated ischemic injury [13]. We recently found that IL-2 upregulates IL-33 receptor (ST2) expression on Tregs, thus enabling IL-33 administration to directly increase Treg and partially protect from IRI by significantly reducing plasma $\mathrm{Cr}$ and tubular injury levels halfway to normal [14]. Based on the observation that IL-2 and IL-33 signal through independent pathways that synergize to increase Tregs and protect mice from AKI, we generated a recombinant cytokine (IL233), bearing IL-2 and IL-33 in a single molecule, which outperformed a simple co-injection of IL-2 and IL-33 for increasing Tregs and protecting from IRI [14]. Importantly, administration of IL233 after IRI completely ameliorated renal injury and mortality that was accompanied by an increase in the ST2 ${ }^{+}$Tregs in kidneys [14]. In another study, treatment with IL233 as late as 2 weeks after doxorubicin-induced AKI restored renal structure and function, as well as prevented fibrosis and mortality [15]. Importantly, treatment with IL233 after the injury induced a regenerative program in the kidneys, as observed by enrichment of tubular progenitors in a Treg-dependent manner [15].

\section{Conclusion}

Despite the major role of inflammation in the pathogenesis of AKI, current treatments do not include antiinflammatory regimen. Tregs offer a mechanism to regulate inflammation, and the evidence for their contribution to tissue repair is growing rapidly. The availability of several potential translational approaches discussed here warrants the utilization of endogenous Tregs for the prevention and treatment of AKI.

\section{Acknowledgements}

The author of this manuscript was supported by LaunchPad Diabetes Fund (R.S.), and National Institute of Diabetes and Kidney Diseases and National Institute of Allergy and Infectious Diseases oftheNIH, underawards R01DK104963(R.S.), R21DK112105 (R.S., M. Rosner and K. Lynch), 1R01DK105833 (R.S. and S.M. $\mathrm{Fu}$ ), and 2R01AI116725 (Subcontract to R.S. from Dr. F. Perrino, Wake Forest University).

\section{Conflict of Interest Statement}

The IL233 cytokine was granted U. S. Patent No. 9,840,545.

\section{References}

1 Sharma R, Kinsey GR. Regulatory T cells in acute and chronic kidney diseases. Am J Physiol Renal Physiol. 2018 May 1;314(5): F679-98.

2 Kinsey GR, Sharma R, Huang L, Li L, Vergis $\mathrm{AL}, \mathrm{Ye} \mathrm{H}$, et al. Regulatory T cells suppress innate immunity in kidney ischemia-reperfusion injury. J Am Soc Nephrol. 2009 Aug; 20(8):1744-53.

3 Gandolfo MT, Jang HR, Bagnasco SM, Ko GJ, Agreda P, Satpute SR, et al. Foxp3+ regulatory $\mathrm{T}$ cells participate in repair of ischemic acute kidney injury. Kidney Int. 2009 Oct;76(7): 717-29.

4 Nguyen MT, Fryml E, Sahakian SK, Liu S, Cantarovich M, Lipman M, et al. Pretransplant recipient circulating CD4+CD127lo/tumor necrosis factor receptor 2+ regulatory $\mathrm{T}$ cells: a surrogate of regulatory $\mathrm{T}$ cell-suppressive function and predictor of delayed and slow graft function after kidney transplantation. Transplantation. 2016 Feb;100(2): 314-24.

5 Wang YM, McRae JL, Robson SC, Cowan PJ, Zhang GY, Hu M, et al. Regulatory T cells participate in CD39-mediated protection from renal injury. Eur J Immunol. 2012 Sep;42(9): 2441-51.
6 Kinsey GR, Huang L, Jaworska K, Khutsishvili $\mathrm{K}$, Becker DA, Ye $\mathrm{H}$, et al. Autocrine adenosine signaling promotes regulatory $\mathrm{T}$ cellmediated renal protection. J Am Soc Nephrol. 2012 Sep;23(9):1528-37.

7 Jaworska K, Ratajczak J, Huang L, Whalen K, Yang M, Stevens BK, et al. Both PD-1 ligands protect the kidney from ischemia reperfusion injury. J Immunol. 2015 Jan 1;194(1):325-33.

8 Chen G, Dong Z, Liu H, Liu Y, Duan S, Liu Y, et al. $\mathrm{mTOR}$ signaling regulates protective activity of transferred CD4+Foxp3+ T cells in repair of acute kidney injury. J Immunol. 2016 Nov 15;197(10):3917-26.

9 Kinsey GR, Huang L, Vergis AL, Li L, Okusa MD. Regulatory T cells contribute to the protective effect of ischemic preconditioning in the kidney. Kidney Int. 2010 May;77(9):77180.

10 Kim MG, Lee SY, Ko YS, Lee HY, Jo SK, Cho $\mathrm{WY}$, et al. CD4+ CD25+ regulatory $\mathrm{T}$ cells partially mediate the beneficial effects of FTY720, a sphingosine-1-phosphate analogue, during ischaemia/reperfusion-induced acute kidney injury. Nephrol Dial Transplant. 2011 Jan;26(1):111-24.
11 Kim H, Lee H, Lee G, Jang H, Kim SS, Yoon $\mathrm{H}$, et al. Phospholipase A2 inhibits cisplatininduced acute kidney injury by modulating regulatory $\mathrm{T}$ cells by the $\mathrm{CD} 206$ mannose receptor. Kidney Int. 2015 Sep;88(3):550-9.

12 Koo TY, Lee JG, Yan JJ, Jang JY, Ju KD, Han $\mathrm{M}$, et al. The $\mathrm{P} 2 \mathrm{X} 7$ receptor antagonist, oxidized adenosine triphosphate, ameliorates renal ischemia-reperfusion injury by expansion of regulatory T cells. Kidney Int. 2017 Aug; 92(2):415-31.

13 Kim MG, Koo TY, Yan JJ, Lee E, Han KH, Jeong JC, et al. IL-2/anti-IL-2 complex attenuates renal ischemia-reperfusion injury through expansion of regulatory T cells. J Am Soc Nephrol. 2013 Oct;24(10):1529-36.

14 Stremska ME, Jose S, Sabapathy V, Huang L, Bajwa A, Kinsey GR, et al. IL233, a novel IL-2 and IL-33 hybrid cytokine, ameliorates renal injury. J Am Soc Nephrol. 2017 Sep;28(9): 2681-93.

15 Sabapathy V, Cheru NT, Corey R, Mohammad S, Sharma R. A novel hybrid cytokine IL233 mediates regeneration following doxorubicin-induced nephrotoxic injury. Sci Rep. 2019 Mar 1;9(1):3215. 